\section{A REPORT OF FORTY-EIGHT NEW CASES OF PARATYPHOID FEVER (TYPE A)}

FRED PROESCHER, M.D.

Pathologist of Allegheny General Hospital AND

JOHN A. RODDY, M.D. PITTSBURG, PA.

The scientific investigations of the last decade have shown that paratyphoid has the same geographical prevalence as typhoid fever. Since Achard and Bensaude, in 1896, described the first two cases of this disease and called it infection paratyphoidique, several hundred cases have been recorded. Soon after Achard and Bensaude's discovery 'Widal and Nobecourt isolated an organism from a post-typhoid abscess which was identical with that described by the former observers. Acting on Gilbert's suggestion, they named it para-coli infection. Later Cushing, Hewlett, Coleman and Buxton, Longcope, Libman and Meltzer reported cases in America.

In Germany, Schottmüller and Curth were the first to recognize cases of paratyphoid fever. Schottmüller was the first to divide paratyphoid bacilli into two groups, based on cultural and agglutination differences. De Feyer and Kayser confirmed the observation of Schottmüller and divided paratyphoid bacilli into two groups, $\mathbf{A}$ and $\mathbf{B}$.

During the last five years a great many analogous observations have been reported, which nearly all belong to Schottmüller's paratyphoid, Type B. Infections with paratyphoid bacilli, Type $\mathrm{A}$, are rare; in Germany, where typhoid cases have been closely studied, only a few cases of para A have occurred.

Netter is the only European who has reported a numher of cases of paratyphoid fever caused by the para $A$ bacillus. He reported seventeen cases, but his observations were made by agglutination tests only; blood cultures were not made. It appears that paratyphoid fever A occurs more frequently in the United States than in Europe.

\section{METHODS OF INVESTIGATION}

Before reporting our findings we wish to describe the methods we employed in making blood cultures and agglutination tests. From May 1, 1907, to May 1, 1908, there were 262 medical patients in the Allegheny General Hospital who had fever or abdominal symptoms or both. From every one of these patients 3 to 5 c.c. of venous blood was taken under aseptic precautions and put in 60 c.c. of a sterile mixture of two parts of calf bile, one part bouillon, 10 per cent. glycerin and 2 per cent. peptone. This was then incubated for fifteen hours at $37 \mathrm{C}$., after which it was inoculated on tubes of agar. The agar was then incubated at $37 \mathrm{C}$. for fifteen hours. At the end of that time it was examined microscopically, and if a motile Gram-negative bacillus was present it was inoculated in milk-sugar bouillon, grape-sugar bouilion, milk and litmus-whey. Eighty-one per cent. were negative, 11 per cent. positive typhoid, and 8 per cent. were para A positive. (For rapid, accurate, clinical work it is only necessary to put the blood in calf bile bouillon, incubate for fifteen hours and then inoculate the above-mentioned culture media with it. In this way it is possible to reach a diagnosis in a period of only thirty hours.)

All agglutination tests were made by Proescher's method $^{1}$; the serum used was obtained from animals which had been inoculated with pure cultures of para $A$ and para $B$ bacilli and subsequently developed an agglutination-titer of 1 to 40,000 .

Morphologically, the paratyphoid bacillus is similar to the typhoid bacillus, but is generally a little shorter and has from eight to ten flagella and possesses a high degree of motility. It stains with the usual anilin dyes and is Gram-negative. It ferments grape-sugar, but not milk-sugar, produces no indol, and does not coagulate milk. The surface growth on agar and gelatin can not be distinguished from typhoid cultures. The cultural distinctions between the two groups of paratyphoid bacilli is that A produces a constantly acid reaction in litmus-whey, the solution always being red, while B first turns litmus-whey red, then blue and continues alkaline. This difference is constant, but the agglutination test with an artificial serum is the most reliable method of differentiation. Para $A$ is not so fatal to mice, guinea-pigs and rabbits as para $B$. The lethal dose of para $A$ is a hondred times more than the lethal dose of para $B$.

The organisms isolated from all our patients were exactly the same, and they were identical with paratyphoid A bacilli obtained from other laboratories for comparison.

A complete report of our laboratory investigations, together with a review of all the cases that have been studied and reported throughout the world, will be published at a later date.

\section{CHARACTERISTICS OF DISEASF}

Etiology.-Season, age and sex play the same part in this disease as in typhoid fever. Most of the cases occur in September and October, but a few in March, April and May. In the 48 cases studied, 17 patients were females and 25 males. From 20 to 30 is the most susceptible period; 75 per cent. of the patients were in the third decade of life; 3 were under 20 , being 15,17 and 19 years old. Ten were between 30 and 40 ; one was 48 , and another, the oldest, was 66 . Six of the forty-two stated that they had previously suffered with typhoid fever. Their statements were probably correct, but we can not be positive.

Immunity.-Typhoid fever does not confer immunity from paratyphoid fever. An attack of paratyphoid fever usually immunizes from subsequent attacks. The period of incubation varies from two days to two weeks. Two persons who accidentally swallowed small amounts of a pure culture became ill, one five days and the other seven days after imbibing.

Onset.-This is not so gradual or insidious as in typhoid fever. Headache, backache, stiff neck, soreness of all the muscles, especially those of the back and legs, unnatural fatigue and anorexia are the initial symptoms in the majority of the cases. Fifteen per cent. of the cases start with acute catarrhal pharyngitis, sore throat, a dry, unproductive cough and chilly sensations. In 22 per cent. of the cases, the onset more closely resembles typhoid fever, the chief symptoms being nausea, vomiting, diarrhea and abdominal tenderness. Headache occurs in nearly all cases; epistaxis less commonly than

1. For Proescher's method of agglutination, see Centralbl. Bakteriol, 1902, abth. I, 400 On account of lack of time, the Bakteriol., 1902 , abth. I, p. 400 . On account of lack of time, the feces were only examined in ten cases; in four of them the para $A$
bacillus was isolated. Methyl-green agar was found to be the best medium for this purpose. Urinary examination was made in fifteen cases, the bacteria only being recovered from one. 
in typhoid; 20 per cent. of the patients complained of diarrhea and 25 per cent. were constipated. The tongue is covered with a thin white fur.

First Week.-Headache is the most constant symptom; toward the end of the week the spleen enlarges and becomes tender and a roseolar rash may appear on the abdomen. Headache usually stops before or early in the second week.

Second Week.-Slight tympany, abdominal distention and constipation or diarrhea occur. In contradistinction to typhoid fever, in this disease the appearance of the tongue is the same throughout the disease. It is moist, covered with a thin white fur and is normal in size and shape. The tip and edges are not clean. It does not become dry, swollen, brown, fissured and sore. From the seventh to tenth day, in most cases, the temperature begins to fall, the appetite returns and convalescence sets in.

Variations.-These are frequent in onset and course. According to the most pronounced symptoms of onset, the cases may be divided into three groups: 1 , those in which nausea, vomiting, diarrhea and abdominai tenderness are the chief symptoms ( 22 per cent. of the cases belong to this group) ; 2 , those in which the predominating symptoms are acute catarrhal pharyngitis, sore throat and cough (these constitute 15 per cent. of the cases); and, 3 , the greatest number, the typical cases, in which the patients complain of severe headache, backache, stiff neck, marked soreness of all the muscles, but especially those of the back and legs and excessive fatigue. The most striking symptom in this group is the stiff neck. In some cases the onset is sudden; in all it is more rapid than in typhoid fever.

Temperature.-The temperature curve was not like the classical one described by Wunderlich. It rose less gradually, sometimes abruptly; the daily fluctuation was greater; the fastigium was much shorter and not so high. On the first, second and third days the evening temperature was $1 \frac{1}{2}$ degrees $F$. higher than the evening temperature of the day before, and each morning there was a remission of a fraction of a degree. The highest temperature was reached the evening of the third or fourth day and was about $103 \mathrm{~F}$. During the next three or five davs the evening temperature maintained the same level; each morning there was a remission of 1 to $1 \frac{1}{2}$ degrees. From the sixth to ninth day lysis began in most cases. The temperature fell a degree every evening and the morning remission was marked; the temperature became normal about the fifteenth day. Hyperpyrexia did not occur and the temperature in itself was never a dangerous symptom. After the temperature had been normal for a week, a relapse might set in and be of shorter, equal or longer duration than the initial attack.

Skin.--In one-third of the cases, those of patients in whom the temperature stayed about $100 \mathrm{~F}$. for more than ten days, a roseolar eruption appeared on the abdomen and one on the chest. It consisted of discrete, circular, elevated, flattened papules, 2 to $4 \mathrm{~mm}$. in diameter, which disappeared on pressure and reappeared when the pressure was removed; they lasted from three to six days and gradually faded away. No other signs appeared on the surface of the body, and in this the disease differed from typhoid fever.

Mouth.-When first observed the tongue was uniformly covered with a white fur, moist, did not become brown, dry, enlarged or fissured nor painful and rapidly cleaned up when the temperature returned to normal. Acute catarrhal pharyngitis occurred in six cases; the mucous membrane was dry, red and injected. Parotid abscess occurred in one case. It was opened, drained and rapidly healed; the pus was not examined.

Respiratory System.-Acute catarrhal laryngitis, cough and bronchitis occurred at the onset in twelve cases. Pneumonia occurred in one case, that of a patient who had been ill in bed at home for three weeks, and was admitted with lobar pneumonia involving both lungs.

Circulatory System.-The pulse was from 80 to 110 , and the pulse-temperature ratio was about the same as in typhoid fever. Dicrotism occurred in only three cases, which was in contrast to typhoid. The pulse was regular and of good tension. After the seventh day the pulse became slightly irregular in some cases. No heart lesion developed during the disease.

Gastrointestinal Tract.-Nausea and vomiting were present at the onset in some cases, but soon disappeared. In many patients the bowels were normal at onset and later became constipated. Unfortunately the stools were not studied. Slight tympany and distention occurred in some cases, but was of short duration. Abdominal pain and tenderness was present at the onset in nine cases. A mild diarrhea, which lasted less than a week, occurred in about 20 per cent. of the cases. The majority of the patients, while in bed, were constipated.

Urinary System.-The variation from normal in the urine was slight. Retention occurred in one case. In 25 per cent. of the cases the Diazo reaction was positive. The urine was examined for bacteria in fifteen cases, but was present in only one.

Liver and Spleen.-The liver was found enlarged in three cases. The area of splenic dulness was increased, the organ not palpable, in ten cases; in fourteen cases the spleen was palpable and tender.

Complications.-These are rare and probably never fatal. One patient developed a parotid abscess and recovered. A patient who had been ill in bed at home for three weeks was admitted with consolidation of both lungs and died in less than twenty-four hours after admission. No one can say whether the pneumonia was a complication of paratyphoid fever or the paratyphoid an occurrence in the course of some other disease.

Prognosis and Mortality.-The prognosis is far more favorable than in typhoid fever, since complications are few. Death is only a possibility. Recovery is rapid and uninterrupted in most cases. The mortality is less than 2 per cent.

\section{CONCLUSION}

Paratyphoid fever is a distinct disease, differentiated from typhoid fever by the following characteristics:

1. It is due to an infection by a bacillus which is not the typhoid bacillus.

2. The onset is more sudden, the duration shorter and the disease milder than typhoid fever.

3. Hyperpyrexia never occurs, complications are rare, and there are no sequelæ.

4. The mortality is less than 2 per cent.

On account of the variability of the initial symptoms, the maintenance of fever for more than two weeks in some cases and the frequent similarity of irregular cases to mild cases of typhoid fever, a positive clinical diagnosis can rarely be made. All require, and should have, for exact diagnosis, an examination of the blood and the recovery, growth and recognition of the bacteria. 
The 48 cases described in this paper occurred in the service of Drs. O.Hail, Wolf and MacNaugher, members of the medical staff Allegheny General Hospital. We wish to express our indebtedness to them for the use of their clinical charts and records.

\section{BIBLIOGRAPHY}

The following articles may be found of interest in this connection :

1. Achard and Bensaude: Infections paratyphoidiques, Bull. et mém. Soc. mêd. đes hôp. de Paris, 1896, vol. xiii, p. 820.

2. Blumenthal: München. med. Wchnschr., 1904, No. 37.

3. Brion and Kayser: Deutsch. Arch. f. klin. Med., 1906 $1 \times x \times v, 525$.

4. Brion and Kayser: München. med. Wchnschr., 1902, No. 15.

5. Buxton and Coleman: New York Med. Jour., 1902, Ixxv, 976.

6. Cushing: New York Med. Jour., 1900, p. 156; Johns Hopkins

IIosp. Bull., 1908.

7. Gwyn, N. B. : Johns Hopkins Hosp. Bull., 1898, ix, 54.

8. Hewlett: Am. Jour. of Med. Sc., 1902, cxxiv, ,200.

9. Johnston: Am. Jour. Med. Sc., August, 1902, cxxiv, 187.

10. Libman, E.: Bacteriologic Study of a Case of Paracolon Infection, Jour. Med, Research, 1902, viil. 168 .

11. Longcope, W. T.: Paracolon Infections, Am. Jour. Med. Sc, August, 1902.

12. Meltzer: New York Med. Jour., 1902, 1xxv, 138.

13. Netter : Compt. rend. Soc., biol., November, 1905.

$\mathrm{xl}, 285$.

14. Schottmüller: Zeitschr. f. Hyg., 1901, Bd. 36

15. Widal: Senaine méd., 1897, p. 333.

16. Widal and Nobecourt:' Semaine med., p. 285.

\section{Therapeutics}

\section{TOXEMIAS OF PREGNANCY}

The disturbances of the last month of pregnancy and some of the severe conditions at parturition are due to varying causes, and the treatment that is efficient and satisfactory in one instance may not be efficient in another.

Dr. Collin Foulkrod, Philadelphia, in the Ameriran Journal of the Medical Sciences, October, 1908, well discusses this important subject.

$\mathrm{He}$ cites the following causes of toxemias due to or during pregnancy: These are intestinal indigestion; disturbances of the parathyroids and thyroid; disturbances in the fetal organism, in the placenta, in the kidneys, or in the liver. He emphasizes the seriousness of changes in the liver, and believes that when metabolic disturbances have involved the liver the patient's condition is serious.

He believes that 50 per cent. of pregnant women suffer from some form of intestinal disturbance, and it is certainly true that intestinal disturbances are concomitant with or preliminary or subsequent to most toxemias.

When instances occur which are positively demonstrable of serious toxemias being caused by prolonged constipation, it is certainly logical to presume that constipation even in a mild form is provocative of the absorption of toxins that should have been eliminated by the intestines. These toxins seriously impair the perfect activity of -the liver, to which they first go through the portal circulation. If the liver is so continuously irritated it can not well do its normal work, and more or less toxins or irritants to metabolism soon get through this filter into the systemic blood and cause nervous, circulatory and kidney irritations. Such irritations at first, and perhaps continuously, may be inconsequential, but they may be the forerunners or the instigators of serious conditions in the latter part of the pregnancy. A primary axiom in pregnancy, then, should be that constipation must not be allowed, and such means must be inaugurated and persisted in as will prevent constipa- tion, intestinal stasis, intestinal fermentation, putrefaction and the absorption of toxins. If any one of the digestive organs is not properly functionating it should be assisted, if possible, and the diet so arranged as not to aggravate this disabled organ, and to relieve it of as much work as possible.

It is now acknowledged, both physiologically and clinically, that the thyroid gland normally hypersecretes during pregnancy. This is doubtless necessary to neutralize the nitrogenous poisons that occur from the double metabolism of mother and child. If the thyroid does not secrete properly, various toxemias occur. A malsecreting thyroid can probably allow pernicious vomiting to occur during pregnancy, can certainly interfere with the nutrition of the fetus, can interfere with the health of the mother, and can allow puerperal eclampsia.

If by a careful study of the pregnant patient it is decided that the thyroid gland is not properly secreting, thyroid substance should be administered. The dose during pregnancy should be small. Just preceding parturition, if it is feared that eclampsia may occur, and especially if the kidneys are insufficient, thyroid should be given in large doses; and if eclampsia does occur, it should be given in very large doses. If a patient gives birth to a child showing imperfect development or symptoms of under-thrroid secretion, although it might not be a cretin, certainly thyroid should be administered to the mother throughout her subsequent pregnancies, unless the symptoms present forbade its use. A daily dose for a pregnant patient, short on thyroid secretion, should not be more than 0.20 gram (3 grains) once a day, while a patient who showed serious toxemia or critical symptoms of metabolic poisoning should receive 0.30 gram (5 grains) three or four times a day for a short period. A patient needing thyroid just before or during parturition may be given 0.60 gram (10 grains) once or twice, while a patient with eclampsia may be given 1.30 grams (20 grains) at one dose.

While Foulkrod speaks of disturbance of the function of the parathyroids being possibly a cause of toxemia, it has not yet been proved. Consequently under what conditions prepared parathyroid gland substance should be administered to the pregnant woman has not been determined, and therefore its administration would be empirical.

Malnutritions of the fetus and degenerations of the placenta may cause the formation and absorption of toxins that will poison the mother. Such toxemias partake of the nature of blood poisoning. The cause being discovered and improvement not soon taking place would seem to call for cleaning out the uterus-this, of course, after a consultation.

Foulkrod does not believe that primary kidney toxemias àre so frequent as primary liver toxemias, but believes that a secondary kidney disturbance may be the consequence of almost any toxemia. This has doubtless many times been the cause for laying the blame of pregnancy toxemias on the kidneys when these organs have not been at fault.

As Foulkrod says, a pure uncomplicated true nephritic toxemia should certainly be discovered. A uremic condition from nephritis of pregnancy must almost always be a progressive condition. Consequently a progressive chronic nephritis can only be overlooked by utterly neglecting to make proper examinations of the urine. If a chronic nephritis, by evidences in the urine, shows a progressive inflammation, a final toxemia, due to allowing a pregnancy to continue too long before interference, 